\title{
Stochastic prey-predator system with foraging arena scheme
}

\author{
Yongmei Cai ${ }^{\mathrm{a}, *}$, Xuerong $\mathrm{Mao}^{\mathrm{a}}$ \\ ${ }^{a}$ Department of Mathematics and Statistics, University of Strathclyde, Glasgow G1 1XH, UK
}

\begin{abstract}
In this paper we extend the foraging arena model describing the dynamics of preypredator abundance from a deterministic framework to a stochastic one. This is achieved by introducing the environmental noises into the growth rate of prey as well as the death rate of predator populations. We then prove that this stochastic differential equation (SDE) has a unique global positive solution. The long-time behaviours of the system are then developed. Furthermore the existence of a stationary distribution is pointed out under certain parametric restrictions. All the results are illustrated by the computer simulations.
\end{abstract}

Keywords: Stochastic prey-predator model, Brownian motion, Extinction, Ultimate boundedness, Stationary distribution

\section{Introduction}

In prey-predator models, the trophic function $\lambda_{2}$ in equation (1.1) links the dynamics of prey and predator populations:

$$
\begin{aligned}
& \frac{d x(t)}{d t}=\lambda_{1}(x(t)) x(t)-\lambda_{2}(x(t), y(t)) y(t) \\
& \frac{d y(t)}{d t}=\gamma \lambda_{2}(x(t), y(t)) y(t)-\lambda_{3}(y(t)) y(t),
\end{aligned}
$$

where $x(t)$ and $y(t)$ represent the population densities of prey and predator at time $t$, $\lambda_{1}(x(t))$ is the per capita net prey growth in absence of predator, $\lambda_{2}(x(t), y(t))$ is the

\footnotetext{
*Corresponding author

Email address: yongmei.cai@strath.ac.uk (Yongmei Cai)
} 
density-dependent uptake response of consumers, $\gamma$ is trophic efficiency ranging from 0 to 1 and $\lambda_{3}(y(t))$ is the consumers death rate. Especially, $\lambda_{2}(x(t), y(t))$ is called the "functional response" in the prey equation (1.1a) and the "numerical response" in the consumers equation (1.1b) [1, 2]. The simplest description of the trophic function $\lambda_{2}(x, y)$ is dependent solely on prey abundance. One is the classic Lotka-Volterra type response in which per capita uptake by the consumers is linearly related to the prey density. Another is Holling Type equation [3]. The Holling II function is widely studied in terrestrial and aquatic food chain models [4] of the form

$$
\lambda_{2}(x)=u_{1} x /\left(u_{2}+x\right),
$$

where $u_{1}$ is a maximum uptake rate by the predator and $u_{2}$ is a prey half-saturation coefficient. An alternative nonlinear formulations of trophic function is the Holling III function:

$$
\lambda_{2}(x)=u_{1} x^{2} /\left(u_{2}^{2}+x^{2}\right) .
$$

In contrast to the prey-dependent uptake response, the trophic function depending on both the prey and consumers abundance suppresses responsiveness by regulating the flux between prey and predator [2]. The simplest form of uptake regulation is ratio dependence $[1,5,6]$ of the form

$$
\lambda_{2}(x, y)=\lambda_{2}(x / y)
$$

However, an extreme property happened to the ratio-dependent formulation is that the uptake rate tends to infinity as consumer abundance tends to zero [7]. Hence the concerned model fails to satisfy the continuity condition at origin. To alleviate this property, the Beddington-DeAngelis type was then proposed by [8, 9]. This type is capable to take care of a number of ecological mechanisms with

$$
\lambda_{2}(x, y)=u_{3} x /\left(u_{4}+u_{5} x+y\right)
$$

where $u_{3} / u_{4}=$ predator capture rate and $u_{5} / u_{4}=$ handling time per prey item [10]. Another functional response to avoid the extreme property happened to the ratio dependence model is the foraging arena model pointed out by [11, 12] with

$$
\lambda_{2}(x, y)=s x /(\beta+y),
$$

where $\beta$ is the consumer density at half maximum per capita uptake rate and $s / \beta$ is the maximum per capita uptake rate by predator. Foraging arenas are common in aquatic systems. They are formed by a series of mechanisms such as restrictions 
of consumer distributions in response to predation risk due to their own predators and the risk-sensitive foraging behaviour by their prey. In foraging arenas, the prey populations are divided into vulnerable and invulnerable population components, with exchange between these two states potentially limit overall trophic flow [12]. The classic Lotka-Volterra model and the Holling type ones assume that the individual prey and predator items are distributed in a spatially uniform way. While the foraging arena model considers the spatial and temporal restrictions in predator and prey activities. Moreover compared to the Beddington-DeAngelis response, the foraging arena model focuses more on the vulnerability exchange of prey abundance, though both models consider a space-limited predation. The foraging arena theory has been widely used in fisheries scientists to explain and model responses of harvested ecosystems. This is done mainly through the application of Ecosim which is the dynamic modelling part of an ecosystem modelling software suite called Ecopath with Ecosim ( $E w E)$. Ecosim is built around foraging arena theory and is capable to fit historical data on responses of multiple fish populations to harvesting and changes in primary production regimes. The key feature of the foraging arena model is equifinality, in the sense that the qualitative model form is robust to lack of information about the cause of limited access to prey and vulnerability exchange [11, 12]. The two-dimensional foraging arena prey-predator model is in a form

$$
\begin{aligned}
& d \bar{x}_{1}(t)=\bar{x}_{1}(t)\left(a-b \bar{x}_{1}(t)-\frac{s \bar{x}_{2}(t)}{\beta+\bar{x}_{2}(t)}\right) d t \\
& d \bar{x}_{2}(t)=\bar{x}_{2}(t)\left(\frac{h \bar{x}_{1}(t)}{\beta+\bar{x}_{2}(t)}-c-f \bar{x}_{2}(t)\right) d t,
\end{aligned}
$$

where $\bar{x}_{1}(t)$ and $\bar{x}_{2}(t)$ represent the population densities of prey and predator in model (1.2) at time $t$ and $a, b, s, \beta, h, c$ and $f$ are all positive constants. $a$ is the intrinsic growth rate of prey, $c$ is the density-dependent mortality rate of consumer, $h=\gamma s, b$ and $f$ are the quadratic mortality rates of prey and predator respectively. We set $\bar{x}(t)=\left(\bar{x}_{1}(t), \bar{x}_{2}(t)\right)^{T}$ as the solution of model (1.2) with the initial value $\bar{x}_{0}=\left(\bar{x}_{1}(0), \bar{x}_{2}(0)\right)^{T}$. In model (1.2), there are two non-negative trivial equilibrium points $\bar{E}_{0}=(0,0)$ and $\bar{E}_{1}=\left(\frac{a}{b}, 0\right)$. Also an unique interior equilibrium point $\bar{E}^{*}\left(\bar{x}_{1}^{*}, \bar{x}_{2}^{*}\right)$ with the nullclines

$$
\begin{aligned}
& \left(a-b \bar{x}_{1}^{*}\right)\left(\beta+\bar{x}_{2}^{*}\right)=s \bar{x}_{2}^{*}, \\
& \left(\beta+\bar{x}_{2}^{*}\right)\left(c+f \bar{x}_{2}^{*}\right)=h \bar{x}_{1}^{*}
\end{aligned}
$$


exists and is globally asymptotically stable provided that $a>\frac{b \beta c}{h}[13]$.

In fact, population systems are always subject to environmental noise, however, the deterministic models do not take environmental changes into account. Hence a natural response to the inevitable variability in the real world would be to consider stochastic model which incorporates some representation of randomness [14, 15]. So far many authors have been studying the population systems under environmental noises described by stochastic differential equations (SDEs). These are obtained by perturbating the parameters in the existing deterministic model system or as scaling limits of individual based model [16]. There have been intense studies on the Lotka-Volterra model (e.g.[15, 17.-19]). Mao et al.[15] revealed an important fact in the Lotka-Volterra model that the environmental noise can suppress a potential population explosion. Takeuchi et al.[20] discussed a surprising effect of colour noise on a Lotka-Volterra model. The stochastic prey-predator systems with Holling II response are also well studied [21-24]. According to Liu et al.[23], the long-time behaviours of the two species and the stationary distribution were explored. Moreover, the more complicated ratio-dependent response and the Beddington-DeAngelis functional response have also been considered by some authors (e.g.[25, 26]). Ji et al.[25] established the conditions for species in a ratio-dependent population system to be either extinct or persistent. In [26], the asymptotic behaviours of the prey-predator system with Beddington-DeAngelis response were investigated and the conditions of having a stationary distribution were produced. However to the best of our knowledge, there has not been enough work about the foraging arena model incorporating the environmental noise. $\mathrm{Ob}$ viously the intrinsic prey growth rate and the consumer death rate in model (1.2) are varied by some environmental factors such as temperature fluctuations and the changes in the composition of the nutrient resource. Suppose that $a$ and $c$ are stochastically perturbed with

$$
a \rightarrow a+\sigma_{1} \dot{B}_{1}(t) \text { and } c \rightarrow c+\sigma_{2} \dot{B}_{2}(t),
$$

where $B_{1}(t)$ and $B_{2}(t)$ are two independent Brownian motions with the intensities represented by two positive constants $\sigma_{1}$ and $\sigma_{2}$. As a result this perturbed system is given by

$$
\begin{aligned}
& d x_{1}(t)=x_{1}(t)\left(a-b x_{1}(t)-\frac{s x_{2}(t)}{\beta+x_{2}(t)}\right) d t+\sigma_{1} x_{1}(t) d B_{1}(t) \\
& d x_{2}(t)=x_{2}(t)\left(\frac{h x_{1}(t)}{\beta+x_{2}(t)}-c-f x_{2}(t)\right) d t-\sigma_{2} x_{2}(t) d B_{2}(t)
\end{aligned}
$$


where $x_{1}(t)$ and $x_{2}(t)$ represent the population densities of prey and predator in model (1.3) at time $t$. We set $x(t)=\left(x_{1}(t), x_{2}(t)\right)^{T}$ as the solution of model (1.3) with the initial value $x_{0}=\left(x_{1}(0), x_{2}(0)\right)^{T}$. Throughout this paper, unless otherwise specified, we let $\left(\Omega,\left\{\mathcal{F}_{t}\right\}_{t \geqslant 0}, \mathbb{P}\right)$ be a complete probability space with a filtration $\left\{\mathcal{F}_{t}\right\}$ satisfying the usual conditions (i.e.it is right continuous and increasing while $\left\{\mathcal{F}_{0}\right\}$ contains all $\mathbb{P}$-null sets). Let $B(t)=\left(B_{1}(t), B_{2}(t)\right)^{T}$ be a two-dimensional Brownian motion defined on this probability space. We denote by $\mathbb{R}_{+}^{2}$ the positive cone in $\mathbb{R}^{2}$, that is $\mathbb{R}_{+}^{2}=\left\{x \in \mathbb{R}^{2}: x_{1}>0\right.$ and $\left.x_{2}>0\right\}$. We also set inf $\emptyset=\infty$. If $\mathrm{A}$ is a vector or matrix, its transpose is denoted by $A^{T}$. If $\mathrm{A}$ is a matrix, its trace norm is $|A|=\sqrt{\operatorname{trace}\left(A^{T} A\right)}$ whilst its operator norm is $\|A\|=\sup \{|A x|:|x|=1\}$. If $\mathrm{A}$ is a symmetric matrix, its smallest and largest eigenvalue are denoted by $\lambda_{\min }(A)$ and $\lambda_{\text {max }}(A)$. Consider the $n$-dimensional stochastic differential equation

$$
d z(t)=\bar{f}(t) d t+\bar{g}(t) d w(t)
$$

for $t \geqslant 0$, where $z(t)=\left(z_{1}(t), \cdots, z_{n}(t)\right)^{T}$ and $w(t)=\left(w_{1}(t), \cdots, w_{n}(t)\right)^{T}$ be an $n$-dimensional Brownian motion defined on the complete probability space $\left(\Omega,\left\{\mathcal{F}_{t}\right\}_{t \geqslant 0}, \mathbb{P}\right)$ adapted to the filtration $\left\{\mathcal{F}_{t}\right\}_{t \geqslant 0}$. Let $C^{2,1}\left(\mathbb{R}^{n} \times \mathbb{R}_{+} ; \mathbb{R}\right)$ be the family of all real-valued functions $V(z, t)$ defined on $\mathbb{R}^{n} \times \mathbb{R}_{+}$such that they are continuously twice differentiable in $z$ and once in $t$. Given $V \in C^{2,1}\left(\mathbb{R}^{n} \times \mathbb{R}_{+} ; \mathbb{R}\right)$, define an operator $L V: \mathbb{R}^{n} \times \mathbb{R}_{+} \rightarrow \mathbb{R}$ by

$$
L V(z, t)=V_{t}(z, t)+V_{z}(z, t) \bar{f}(t)+\frac{1}{2} \operatorname{trace}\left(\bar{g}^{T}(t) V_{z z}(z, t) \bar{g}(t)\right),
$$

which is called the diffusion operator of the Itô process (1.4) associated with the $C^{2,1}$-function $V$ (see e.g.[27, p. 41]). With the diffusion operator, the Itô formula (1.4) can be written as

$$
d V(z(t), t)=L V(z(t), t) d t+V_{z}(z(t), t) \bar{g}(t) d w(t) \quad \text { a.s. }
$$

In this paper, we first verify the existence of a positive global solution of model (1.3) in section 2. Next we explore the asymptotic moment average of model system in section 3. Moreover in the following section we give the parametric conditions for the system to be extinct. Finally the stationary distribution of system $(1.3)$ is examined in section 5 .

\section{Global positive solution}

To investigate the dynamical behaviour of model $(1.3)$, the existence of a unique global positive solution is verified first. The coefficients of the SDE model 
(1.3) are locally Lipschitz continuous, however, they do not satisfy linear growth condition (see eg.[28, pp. 51, 56-57] for more details). Hence the existing general existence-and-uniqueness theorem on SDEs is not applicable to model (1.3) and there exists a unique maximal local solution to model (1.3). That is, the solution may exit from $\mathbb{R}_{+}^{2}$ space at a finite time [15, 27]. In this section, we shall show that the solution of model $(1.3)$ is positive and global as in [15, 29].

Theorem 2.1. For any given initial value $x_{0} \in \mathbb{R}_{+}^{2}$, there is a unique solution $x(t)$ to equation $(1.3)$ on $t \geqslant 0$ and the solution will remain in $\mathbb{R}_{+}^{2}$ with probability 1 , namely $x(t) \in \mathbb{R}_{+}^{2}$ for all $t \geqslant 0$ almost surely.

Proof. Since the coefficients of the equation (1.3) are locally Lipschitz continuous, for any given initial value $x_{0} \in \mathbb{R}_{+}^{2}$, there is a unique maximal local solution $x(t)$ on $t \in\left[0, \tau_{e}\right.$ ), where $\tau_{e}$ is the explosion time (exit time) from $\mathbb{R}_{+}^{2}$. To show that this solution is global, we need to verify $\tau_{e}=\infty$ a.s. Let $k_{0}>0$ be sufficiently large for $x_{1}(0)$ and $x_{2}(0)$ lying within the interval $\left[\frac{1}{k_{0}}, k_{0}\right]$. For each integer $k \geqslant k_{0}$, define the stopping time

$$
\tau_{k}=\inf \left\{t \in\left[0, \tau_{e}\right): x_{i}(t) \notin\left(\frac{1}{k}, k\right) \text { for } i=1,2\right\} .
$$

$\tau_{k}$ is increasing as $k \rightarrow \infty$. Set $\tau_{\infty}:=\lim _{t \rightarrow \infty} \tau_{k}$ and whence $\tau_{\infty} \leqslant \tau_{e}$ a.s. Hence to complete the proof, we need to show that

$$
\tau_{\infty}=\infty \quad \text { a.s. }
$$

If (2.1) is not true, there are three constants $T>0, k_{1} \geqslant k_{0}$ and $\epsilon \in(0,1)$ such that

$$
\mathbb{P}\left(\Omega_{k}\right) \geqslant \epsilon \text { for all } k \geqslant k_{1} \text {, where } \Omega_{k}=\left\{\tau_{k} \leqslant T\right\} .
$$

Define a $C^{2}$-function $V: \mathbb{R}_{+}^{2} \rightarrow \mathbb{R}_{+}$by $V(x)=x_{1}-\log x_{1}+x_{2}-\log x_{2}$. From the Itô formula,

$$
d V(x(t))=L V(x(t)) d t+\left(\sigma_{1} x_{1}(t)-\sigma_{1}\right) d B_{1}(t)-\left(\sigma_{2} x_{2}(t)-\sigma_{2}\right) d B_{2}(t),
$$

where

$$
\begin{aligned}
L V(x)= & -a+\frac{s x_{2}}{\beta+x_{2}}+c+\frac{\sigma_{1}^{2}}{2}+\frac{\sigma_{2}^{2}}{2}+(a+b) x_{1}+\frac{h x_{1} x_{2}}{\beta+x_{2}}+(f-c) x_{2}-\frac{s x_{1} x_{2}}{\beta+x_{2}} \\
& -\frac{h x_{1}}{\beta+x_{2}}-b x_{1}^{2}-f x_{2}^{2} \\
\leqslant & -a+s+c+\frac{\sigma_{1}^{2}}{2}+\frac{\sigma_{2}^{2}}{2}+(a+b+h) x_{1}+(f-c) x_{2}-b x_{1}^{2}-f x_{2}^{2},
\end{aligned}
$$


which is bounded, say by $Q$, in $\mathbb{R}_{+}^{2}$. Consequently,

$$
\mathbb{E} V\left(x\left(\tau_{k} \wedge T\right)\right) \leqslant V\left(x_{0}\right)+Q \mathbb{E}\left(\tau_{k} \wedge T\right) \leqslant V\left(x_{0}\right)+Q T .
$$

Moreover for all $\omega \in \Omega_{k}, x_{1}\left(\tau_{k}, \omega\right)$ or $x_{2}\left(\tau_{k}, \omega\right)$ equals either $k$ or $\frac{1}{k}$. Hence

$$
V\left(x\left(\tau_{k}\right)\right) \geqslant(k-\log k) \wedge\left(\sqrt{\frac{1}{k}}+\log k\right) .
$$

This with 2.2 infers

$$
V\left(x_{0}\right)+Q T \geqslant \mathbb{E}\left(I_{\Omega_{k}} V\left(x\left(\tau_{k}\right)\right)\right) \geqslant \epsilon\left((k-\log k) \wedge\left(\sqrt{\frac{1}{k}}+\log k\right)\right) .
$$

This leads to a contradiction as we let $k \rightarrow \infty$,

$$
\infty>V\left(x_{0}\right)+Q T=\infty .
$$

So we have $\tau_{\infty}=\infty$ a.s.

\section{Asymptotic moment estimate}

After analysing the global positive solution of model (1.3), we now explore the long-time dynamical behaviours of the prey and predator populations.

Theorem 3.1. For any $\theta>0$, there exists a positive constant $K(\theta)$ such that for any initial value $x_{0} \in \mathbb{R}_{+}^{2}$,

$$
\limsup _{t \rightarrow \infty} \mathbb{E}|x(t)|^{\theta} \leqslant K(\theta)
$$

Proof. Applying the Itô formula to $e^{\eta t}\left(x_{1}^{\theta}+x_{2}^{\theta}\right)$ for any $\eta>0$ and $\theta>0$,

$$
\begin{aligned}
e^{\eta t}\left(x_{1}^{\theta}(t)+x_{2}^{\theta}(t)\right) & =x_{1}^{\theta}(0)+x_{2}^{\theta}(0)+\int_{0}^{t} e^{\eta s} f(x(s)) d s+\theta \sigma_{1} \int_{0}^{t} e^{\eta s} x_{1}^{\theta}(s) d B_{1}(s) \\
& -\theta \sigma_{2} \int_{0}^{t} e^{\eta s} x_{2}^{\theta}(s) d B_{2}(s),
\end{aligned}
$$

where

$$
\begin{aligned}
f(x) & =\left(a \theta+\frac{1}{2} \theta(\theta-1) \sigma_{1}^{2}+\eta\right) x_{1}^{\theta}+\left(-c \theta+\frac{1}{2} \theta(\theta-1) \sigma_{2}^{2}+\eta\right) x_{2}^{\theta}-\frac{s \theta x_{1}^{\theta} x_{2}}{\beta+x_{2}} \\
& +\frac{h \theta x_{1} x_{2}^{\theta}}{\beta+x_{2}}-b \theta x_{1}^{\theta+1}-f \theta x_{2}^{\theta+1} .
\end{aligned}
$$


For $\theta \geqslant 1$, the Young inequality yields

$$
\frac{x_{1} x_{2}^{\theta}}{\beta+x_{2}} \leqslant x_{1} x_{2}^{\theta-1} \leqslant \frac{x_{1}^{\theta}}{\theta}+\frac{\theta-1}{\theta} x_{2}^{\theta}
$$

Hence

$$
\begin{aligned}
f(x) & \leqslant\left(a \theta+\frac{1}{2} \theta(\theta-1) \sigma_{1}^{2}+h+\eta\right) x_{1}^{\theta}+\left((\theta-1) h-c \theta+\frac{1}{2} \theta(\theta-1) \sigma_{2}^{2}+\eta\right) x_{2}^{\theta} \\
& -b \theta x_{1}^{\theta+1}-f \theta x_{2}^{\theta+1}
\end{aligned}
$$

which is bounded, say by $K^{*}(\theta)$. Moreover, it follows from (3.1) that

$$
\mathbb{E}\left[e^{\eta\left(t \wedge \tau_{k}\right)}\left(x_{1}^{\theta}\left(t \wedge \tau_{k}\right)+x_{2}^{\theta}\left(t \wedge \tau_{k}\right)\right)\right] \leqslant x_{1}^{\theta}(0)+x_{2}^{\theta}(0)+K^{*}(\theta) \int_{0}^{t \wedge \tau_{k}} e^{\eta s} d s
$$

Letting $k \rightarrow \infty$ and then $t \rightarrow \infty$ yields

$$
\limsup _{t \rightarrow \infty} \mathbb{E}\left[x_{1}^{\theta}(t)+x_{2}^{\theta}(t)\right] \leqslant \lim _{t \rightarrow \infty} \frac{1}{e^{\eta t}}\left(x_{1}^{\theta}(0)+x_{2}^{\theta}(0)+\frac{K^{*}(\theta)\left(e^{\eta t}-1\right)}{\eta}\right)=\frac{K^{*}(\theta)}{\eta} .
$$

On the other hand, we have

$$
|x|^{2} \leqslant 2 \max \left(x_{1}^{2}, x_{2}^{2}\right), \text { so }|x|^{\theta} \leqslant 2^{\theta / 2} \max \left(x_{1}^{\theta}, x_{2}^{\theta}\right) \leqslant 2^{\theta / 2}\left(x_{1}^{\theta}+x_{2}^{\theta}\right) .
$$

As a result,

$$
\limsup _{t \rightarrow \infty} \mathbb{E}|x(t)|^{\theta} \leqslant 2^{\theta / 2} \limsup _{t \rightarrow \infty} \mathbb{E}\left[x_{1}^{\theta}(t)+x_{2}^{\theta}(t)\right] \leqslant \frac{2^{\theta / 2} K^{*}(\theta)}{\eta}=K(\theta) .
$$

For $0<\theta<1$, H ölder's inequality yields

$$
\mathbb{E}|x|^{\theta} \leqslant(\mathbb{E}|x|)^{\theta}
$$

Hence from (3.2)

$$
\underset{t \rightarrow \infty}{\limsup } \mathbb{E}|x(t)|^{\theta} \leqslant \limsup _{t \rightarrow \infty}(\mathbb{E}|x(t)|)^{\theta} \leqslant K(\theta) .
$$




\section{Extinction}

In this section, we investigate the conditions for the system to be extinct.

Lemma 4.1. A one-dimensional Brownian motion $\{W(t)\}_{t \geqslant 0}$ has the property that for almost every $\omega \in \Omega$,

$$
\lim _{t \rightarrow \infty} \frac{\min _{0 \leqslant u \leqslant t} W(u, \omega)}{t}=\lim _{t \rightarrow \infty} \frac{\max _{0 \leqslant u \leqslant t} W(u, \omega)}{t}=0 .
$$

Proof. According to [28], for any $\epsilon>0$, there exists a positive random variable $\rho_{\epsilon}$ such that for almost every $\omega \in \Omega$,

$$
-(1+\epsilon) \sqrt{2 t \log \log t} \leqslant W(t, \omega) \leqslant(1+\epsilon) \sqrt{2 t \log \log t} \quad \text { for all } t \geqslant \rho_{\epsilon}(\omega) .
$$

It then follows that for almost every $\omega \in \Omega$,

$$
\max _{0 \leqslant u \leqslant t} W(u, \omega) \leqslant \max _{0 \leqslant u \leqslant \rho_{\epsilon}(\omega)} W(u, \omega)+(1+\epsilon) \sqrt{2 t \log \log t} .
$$

Letting $t \rightarrow \infty$,

$$
0=W(0) \leqslant \lim _{t \rightarrow \infty} \frac{\max _{0 \leqslant u \leqslant t} W(u)}{t} \leqslant 0 \quad \text { a.s. }
$$

Hence we obtain

$$
\lim _{t \rightarrow \infty} \frac{\max _{0 \leqslant u \leqslant t} W(u)}{t}=0 \quad \text { a.s. }
$$

Similarly, we also have

$$
\lim _{t \rightarrow \infty} \frac{\min _{0 \leqslant u \leqslant t} W(u)}{t}=0 \quad \text { a.s. }
$$

Theorem 4.2. For any initial value $x_{0} \in \mathbb{R}_{+}^{2}$,

(a) if

$$
2 a<\sigma_{1}^{2}
$$

both $x_{1}(t)$ and $x_{2}(t)$ tend to zero exponentially as $t \rightarrow \infty$ with probability 1 ;

(b) if

$$
\sigma_{1}^{2}<2 a<\phi
$$


where

$$
\phi=\sigma_{1}^{2}+\frac{2 b \beta c}{h}+\frac{b \beta \sigma_{2}^{2}}{h}
$$

$x_{1}(t)$ obeys

$$
\lim _{t \rightarrow \infty} \frac{1}{t} \int_{0}^{t} x_{1}(u) d u=\frac{2 a-\sigma_{1}^{2}}{2 b} \quad \text { a.s. }
$$

and $x_{2}(t)$ tends to zero exponentially as $t \rightarrow \infty$ with probability 1.

Proof. (a) Applying the Itô formula on $\log x_{1}$, we have

$$
\begin{aligned}
d \log x_{1}(t) & =\left(a-b x_{1}(t)-\frac{\sigma_{1}^{2}}{2}-\frac{s x_{2}(t)}{\beta+x_{2}(t)}\right) d t+\sigma_{1} d B_{1}(t) \\
& \leqslant\left(a-\frac{\sigma_{1}^{2}}{2}\right) d t+\sigma_{1} d B_{1}(t) .
\end{aligned}
$$

Integrating from 0 to $t$ and dividing by $t$, we get

$$
\frac{1}{t} \log x_{1}(t) \leqslant \frac{1}{t} \log x_{1}(0)+a-\frac{\sigma_{1}^{2}}{2}+\frac{\sigma_{1} B_{1}(t)}{t} .
$$

Letting $t \rightarrow \infty$ and by the large number theorem

$$
\lim _{t \rightarrow \infty} \frac{\sigma_{1} B_{1}(t)}{t}=0 \quad \text { a.s. }
$$

and thus from condition 4.2

$$
\limsup _{t \rightarrow \infty} \frac{1}{t} \log x_{1}(t) \leqslant a-\frac{\sigma_{1}^{2}}{2}<0 \quad \text { a.s. }
$$

as required. Hence $x_{1}(t)$ tends to zero exponentially as $t \rightarrow \infty$ and

$$
\lim _{t \rightarrow \infty} \frac{1}{t} \int_{0}^{t} x_{1}(u) d u=0 \quad \text { a.s. }
$$

Meanwhile

$$
d \log x_{2}(t)=\left(\frac{h x_{1}(t)}{\beta+x_{2}(t)}-c-\frac{\sigma_{2}^{2}}{2}-f x_{2}(t)\right) d t-\sigma_{2} d B_{2}(t) .
$$


It follows that

$$
\frac{\log x_{2}(t)}{t} \leqslant \frac{1}{t}\left(\log x_{2}(0)+\frac{h}{\beta} \int_{0}^{t} x_{1}(u) d u\right)-\left(c+\frac{\sigma_{2}^{2}}{2}\right)-\frac{\sigma_{2} B_{2}(t)}{t} .
$$

Letting $t \rightarrow \infty$ and recalling equation 4.6,

$$
\limsup _{t \rightarrow \infty} \frac{\log x_{2}(t)}{t} \leqslant-\left(c+\frac{\sigma_{2}^{2}}{2}\right)<0 \quad \text { a.s. }
$$

(b) Applying Itô's formula on $\frac{1}{x_{1}}$ gives

$$
d\left(\frac{1}{x_{1}(t)}\right)=\left(\frac{1}{x_{1}(t)}\left(\frac{s x_{2}(t)}{\beta+x_{2}(t)}-a+\sigma_{1}^{2}\right)+b\right) d t-\frac{\sigma_{1}}{x_{1}(t)} d B_{1}(t) .
$$

Hence by the variation-of-constants formula (see e.g. [28, pp. 98-99]),

$$
\begin{aligned}
\frac{1}{x_{1}(t)} & =\exp \left(\int_{0}^{t}\left(\frac{1}{2} \sigma_{1}^{2}-a+\frac{s x_{2}(u)}{\beta+x_{2}(u)}\right) d u-\sigma_{1} B_{1}(t)\right) \\
& {\left[\frac{1}{x_{1}(0)}+b \int_{0}^{t} \exp \left(\int_{0}^{u}\left(a-\frac{s x_{2}(v)}{\beta+x_{2}(v)}-\frac{1}{2} \sigma_{1}^{2}\right) d v+\sigma_{1} B_{1}(u)\right) d u\right] } \\
& =\exp \left(-\sigma_{1} B_{1}(t)\right)\left[\frac{1}{x_{1}(0)} \exp \left(-\left(a-\frac{1}{2} \sigma_{1}^{2}\right) t+s \int_{0}^{t} \frac{x_{2}(u)}{\beta+x_{2}(u)} d u\right)\right. \\
& \left.+b \int_{0}^{t} \exp \left(-\left(a-\frac{\sigma_{1}^{2}}{2}\right)(t-u)+s \int_{u}^{t} \frac{x_{2}(v)}{\beta+x_{2}(v)} d v+\sigma_{1} B_{1}(u)\right) d u\right]
\end{aligned}
$$

On the one hand, (4.8) leads to

$$
\begin{aligned}
\frac{1}{x_{1}(t)} \leqslant & \exp \left(-\sigma_{1} B_{1}(t)\right)\left[\frac{1}{x_{1}(0)} \exp \left(-\left(a-\frac{1}{2} \sigma_{1}^{2}\right) t+s \int_{0}^{t} \frac{x_{2}(u)}{\beta+x_{2}(u)} d u\right)\right. \\
+ & \left.b \exp \left(\sigma_{1} \max _{0 \leqslant u \leqslant t} B_{1}(u)+s \int_{0}^{t} \frac{x_{2}(u)}{\beta+x_{2}(u)} d u\right) \int_{0}^{t} \exp \left(-\left(a-\frac{\sigma_{1}^{2}}{2}\right)(t-u)\right) d u\right] \\
\leqslant & \exp \left(\sigma_{1}\left(\max _{0 \leqslant u \leqslant t} B_{1}(u)-B_{1}(t)\right)+s \int_{0}^{t} \frac{x_{2}(u)}{\beta+x_{2}(u)} d u\right) \\
& {\left[\frac{1}{x_{1}(0)} \exp \left(-\left(a-\frac{1}{2} \sigma_{1}^{2}\right) t\right)+b \int_{0}^{t} \exp \left(-\left(a-\frac{\sigma_{1}^{2}}{2}\right)(t-u)\right) d u\right] } \\
= & \exp \left(\sigma_{1}\left(\max _{0 \leqslant u \leqslant t} B_{1}(u)-B_{1}(t)\right)+s \int_{0}^{t} \frac{x_{2}(u)}{\beta+x_{2}(u)} d u\right) \\
& {\left[\frac{1}{x_{1}(0)} \exp \left(-\left(a-\frac{1}{2} \sigma_{1}^{2}\right) t\right)+\frac{2 b\left(1-\exp \left(-\left(a-\frac{\sigma_{1}^{2}}{2}\right) t\right)\right)}{2 a-\sigma_{1}^{2}}\right] }
\end{aligned}
$$


It follows that

$$
\frac{\log x_{1}(t)}{t} \geqslant-\frac{\log K_{1}(t)}{t}-\frac{\sigma_{1}\left(\max _{0 \leqslant u \leqslant t} B_{1}(u)-B_{1}(t)\right)}{t}-\frac{s}{t} \int_{0}^{t} \frac{x_{2}(u)}{\beta+x_{2}(u)} d u
$$

where

$$
K_{1}(t)=\frac{1}{x_{1}(0)} \exp \left(-\left(a-\frac{1}{2} \sigma_{1}^{2}\right) t\right)+\frac{2 b\left(1-\exp \left(-\left(a-\frac{\sigma_{1}^{2}}{2}\right) t\right)\right)}{2 a-\sigma_{1}^{2}}
$$

and $\sup _{0 \leqslant t<\infty} K_{1}(t)<\infty$ if condition 4.3 holds. By 4.5 and 4.9,

$$
\begin{aligned}
& \frac{1}{t} \int_{0}^{t} x_{1}(u) d u \\
= & \frac{2 a-\sigma_{1}^{2}}{2 b}-\frac{\log x_{1}(t)}{b t}+\frac{\log x_{1}(0)}{b t}-\frac{s}{b t} \int_{0}^{t} \frac{x_{2}(u)}{\beta+x_{2}(u)} d u+\frac{\sigma_{1}}{b t} B_{1}(t) \\
\leqslant & \frac{2 a-\sigma_{1}^{2}}{2 b}+\frac{\log K_{1}(t)}{b t}+\frac{\sigma_{1}\left(\max _{0 \leqslant u \leqslant t} B_{1}(u)-B_{1}(t)\right)}{b t}+\frac{\log x_{1}(0)}{b t}+\frac{\sigma_{1}}{b t} B_{1}(t) .
\end{aligned}
$$

As $t \rightarrow \infty$ and from the large number theorem and Lemma 4.1 .

$$
\limsup _{t \rightarrow \infty} \frac{1}{t} \int_{0}^{t} x_{1}(u) d u \leqslant \frac{2 a-\sigma_{1}^{2}}{2 b} \text { a.s. }
$$

From equation (4.7),

$$
d \log x_{2}(t) \leqslant\left(\frac{h x_{1}(t)}{\beta}-c-\frac{\sigma_{2}^{2}}{2}\right) d t-\sigma_{2} d B_{2}(t) .
$$

This and (4.11) yield

$$
\begin{aligned}
\limsup _{t \rightarrow \infty} \frac{1}{t} \log \left(x_{2}(t)\right) & \leqslant \frac{h}{\beta} \limsup _{t \rightarrow \infty} \frac{1}{t} \int_{0}^{t} x_{1}(u) d u-\left(c+\frac{\sigma_{2}^{2}}{2}\right) \\
& \leqslant \frac{h\left(2 a-\sigma_{1}^{2}\right)}{2 \beta b}-\left(c+\frac{\sigma_{2}^{2}}{2}\right)<0,
\end{aligned}
$$

by condition 4.3 . Hence for arbitrary small $\zeta>0$, there exists $t_{\zeta}$ such that

$$
\mathbb{P}(\bar{\Omega}) \geqslant 1-\zeta \text { where } \bar{\Omega}=\left\{\omega: \frac{s x_{2}(t, \omega)}{b\left(\beta+x_{2}(t, \omega)\right)} \leqslant \zeta \text { for } t \geqslant t_{\zeta}\right\}
$$


On the other hand, (4.8) yields

$$
\begin{aligned}
\frac{1}{x_{1}(t)} & \geqslant \exp \left(-\sigma_{1} B_{1}(t)\right)\left[\frac{1}{x_{1}(0)} \exp \left(-\left(a-\frac{1}{2} \sigma_{1}^{2}\right) t\right)\right. \\
& \left.+b \exp \left(\sigma_{1} \min _{0 \leqslant u \leqslant t} B_{1}(u)\right) \int_{0}^{t} \exp \left(-\left(a-\frac{\sigma_{1}^{2}}{2}\right)(t-u)\right) d u\right] \\
& \geqslant \exp \left(\sigma_{1}\left(\min _{0 \leqslant u \leqslant t} B_{1}(u)-B_{1}(t)\right)\right)\left[\frac{1}{x_{1}(0)} \exp \left(-\left(a-\frac{1}{2} \sigma_{1}^{2}\right) t\right)\right. \\
& \left.+\frac{2 b\left(1-\exp \left(-\left(a-\frac{\sigma_{1}^{2}}{2}\right) t\right)\right)}{2 a-\sigma_{1}^{2}}\right] .
\end{aligned}
$$

Then

$$
\frac{\log x_{1}(t)}{t} \leqslant-\frac{\log K_{1}(t)}{t}-\frac{\sigma_{1}\left(\min _{0 \leqslant u \leqslant t} B_{1}(u)-B_{1}(t)\right)}{t} .
$$

Hence we obtain from 4.10 that

$$
\begin{aligned}
\frac{1}{t} \int_{0}^{t} x_{1}(u) d u & \geqslant \frac{2 a-\sigma_{1}^{2}}{2 b}+\frac{\log K_{1}(t)}{b t}+\frac{\sigma_{1}\left(\min _{0 \leqslant u \leqslant t} B_{1}(u)-B_{1}(t)\right)}{b t}+\frac{\log x_{1}(0)}{b t} \\
& -\frac{s}{b t} \int_{0}^{t} \frac{x_{2}(u)}{\beta+x_{2}(u)} d u+\frac{\sigma_{1}}{b t} B_{1}(t)
\end{aligned}
$$

For any $\omega \in \bar{\Omega},(4.12)$ together with Lemma 4.1 indicates

$$
\liminf _{t \rightarrow \infty} \frac{1}{t} \int_{0}^{t} x_{1}(u) d u \geqslant \frac{2 a-\sigma_{1}^{2}}{2 b}-\zeta .
$$

This and (4.11) implies

$$
\lim _{t \rightarrow \infty} \frac{1}{t} \int_{0}^{t} x_{1}(u) d u=\frac{2 a-\sigma_{1}^{2}}{2 b} \text { a.s. }
$$

Remark 4.3. The parametric restriction for model (1.2) to die out is immediately obtained by setting $\sigma_{1}=\sigma_{2}=0$ in condition (4.3). That is, under condition $0<a<\frac{b \beta c}{h}$, the solution of model (1.2) obeys $\lim _{t \rightarrow \infty} \frac{1}{t} \int_{0}^{t} \bar{x}_{1}(u) d u=\frac{a}{b}$ and the consumers tend to extinction ultimately. 
Theorem 4.2 (a) suggests that both species in model (1.3) will die out if $2 a<$ $\sigma_{1}^{2}$. That is, large white noise intensity $\sigma_{1}^{2}$ can cause the population extinction of both species. In the real life, this may happen when a serious epidemic or severe weather occurs. Notice that this case is not considered by model (1.2). Additionally, from Remark 4.3, the deterministic model (1.2) will die out if $a<$ $\frac{b \beta c}{h}$. In the contrast, due to the existence of white noises, Theorem 4.2 (b) points out that model (1.3) tends to extinction even for some $a>\frac{b \beta c}{h}$ (but need to obey $2 a<\phi)$. In the next section, we examine how the population system behaves when $2 a$ gets even larger.

\section{Stationary distribution}

In this section, we give the conditions for the solution of SDE model (1.3) having a unique stationary distribution. Let $P_{x_{0}, t}$ denote the probability measure induced by $x(t)$ with initial value $x(0)=x_{0}$, that is

$$
P_{x_{0}, t}(F)=\mathbb{P}\left(x(t) \in F \mid x(0)=x_{0}\right)=\mathbb{P}_{x_{0}}(x(t) \in F), \quad F \in \mathcal{B}\left(\mathbb{R}_{+}^{2}\right),
$$

where $\mathcal{B}\left(\mathbb{R}_{+}^{2}\right)$ is the $\sigma$-algebra of all the Borel sets $F \subseteq \mathbb{R}_{+}^{2}$. If there is a probability measure $\mu(\cdot)$ on the measurable space $\left(\mathbb{R}_{+}^{2}, \mathcal{B}\left(\mathbb{R}_{+}^{2}\right)\right)$ such that

$$
P_{x_{0}, t}(\cdot) \rightarrow \mu(\cdot) \text { in distribution for any } x_{0} \in \mathbb{R}_{+}^{2},
$$

we then say that the SDE model (1.3) has a stationary distribution $\mu(\cdot)[27,30,31]$. To show the existence of a stationary distribution, let us first cite a known result from Khasminskii [30, pp. 107-109, Theorem 4.1] as a lemma.

Lemma 5.1. The SDE model (1.3) has a unique stationary distribution if there is a bounded open set $G$ of $\mathbb{R}_{+}^{2}$ such that $\sup _{x_{0} \in Q-G} \mathbb{E}_{x_{0}}\left(\tau_{G}\right)<\infty$ for every compact subset $Q$ of $\mathbb{R}_{+}^{2}$ such that $G \subset Q$ where $\tau_{G}=\inf \{t \geqslant 0: x(t) \in G\}$.

In the original Khasminskii theorem, there is one more condition that

$$
\inf _{x \in G} \lambda_{\min }\left(\operatorname{diag}\left(\sigma_{1}^{2} x_{1}^{2}, \sigma_{2}^{2} x_{2}^{2}\right)\right)>0 \text { for } x \in \mathbb{R}_{+}^{2},
$$

which is obvious and hence there is no point to state.

Theorem 5.2. If

$$
2 a\left(1-\frac{\sigma_{2}^{2}}{2 h}-\frac{c}{h}\right)>\phi
$$

where $\phi$ is denoted in (4.4), then for any initial value $x_{0} \in \mathbb{R}_{+}^{2}$, model (1.3) has a unique stationary distribution. 
Proof. We define a $C^{2}$-function $V: \mathbb{R}_{+}^{2} \rightarrow \mathbb{R}_{+}$:

$$
V(x)=M V_{1}(x)+V_{2}(x)+e,
$$

where

$$
V_{1}(x)=\log \left(\beta+x_{1}\right)-\log \left(x_{1}\right)+\frac{l}{h} x_{2}-\frac{a+b \beta}{h} \log x_{2}, \quad V_{2}(x)=x_{1}+\frac{s}{h} x_{2},
$$

and $e, l$ and $M$ are three constants. $e=-\min \left(M V_{1}(x)+V_{2}(x)\right)$ to keep the nonnegativity of $V(x)$,

$$
l=\left(\frac{h s}{c \beta}+\frac{(a+b \beta) f}{c}\right) \bigvee \frac{(a+b \beta) h}{4 f \beta^{2}}
$$

and $M$ is to be defined later. First compute

$$
\begin{aligned}
L V_{1} & =\left(\frac{x_{1}}{\beta+x_{1}}-1\right)\left(a-b x_{1}-\frac{s x_{2}}{\beta+x_{2}}\right)+\frac{1}{2}\left(\frac{1}{x_{1}^{2}}-\frac{1}{\left(\beta+x_{1}\right)^{2}}\right) \sigma_{1}^{2} x_{1}^{2} \\
& +\left(\frac{l x_{2}}{h}-\frac{a+b \beta}{h}\right)\left(\frac{h x_{1}}{\beta+x_{2}}-c-f x_{2}\right)+\frac{a+b \beta}{2 h} \sigma_{2}^{2} \\
& \leqslant \frac{a x_{1}}{\beta+x_{1}}-\frac{b x_{1}^{2}}{\beta+x_{1}}+b x_{1}-\frac{a+b \beta}{\beta+x_{2}} x_{1}-a+\frac{s x_{2}}{\beta+x_{2}}+\frac{\sigma_{1}^{2}}{2}+\frac{l x_{1} x_{2}}{\beta+x_{2}} \\
& -\frac{c l x_{2}}{h}-\frac{f l x_{2}^{2}}{h}+\frac{(a+b \beta) c}{h}+\frac{(a+b \beta) f}{h} x_{2}+\frac{a+b \beta}{2 h} \sigma_{2}^{2} \\
& \leqslant \frac{a x_{1}}{\beta+x_{1}}+\frac{b \beta x_{1}}{\beta+x_{1}}-\frac{a+b \beta}{\beta+x_{2}} x_{1}-a+\frac{\sigma_{1}^{2}}{2}+\frac{a+b \beta}{h} c+\frac{a+b \beta}{2 h} \sigma_{2}^{2} \\
& +\left(\frac{s}{\beta}+\frac{(a+b \beta) f}{h}-\frac{c l}{h}\right) x_{2}-\frac{f l}{h} x_{2}^{2}+\frac{l x_{1} x_{2}}{\beta+x_{2}} \\
& =(a+b \beta) x_{1} \frac{x_{2}-x_{1}}{\left(\beta+x_{1}\right)\left(\beta+x_{2}\right)}-\lambda+\left(\frac{s}{\beta}+\frac{(a+b \beta) f}{h}-\frac{c l}{h}\right) x_{2}-\frac{f l}{h} x_{2}^{2}+\frac{l x_{1} x_{2}}{\beta+x_{2}} \\
& \leqslant \frac{a+b \beta}{\beta^{2}}\left(x_{1} x_{2}-x_{1}^{2}\right)-\lambda+\left(\frac{s}{\beta}+\frac{(a+b \beta) f}{h}-\frac{c l}{h}\right) x_{2}-\frac{f l}{h} x_{2}^{2}+\frac{l x_{1} x_{2}}{\beta+x_{2}},
\end{aligned}
$$

where $\lambda=a-\frac{\sigma_{1}^{2}}{2}-\frac{a+b \beta}{h} c-\frac{a+b \beta}{2 h} \sigma_{2}^{2}>0$ from condition 5.1. By the Young inequality and (5.2),

$$
L V_{1} \leqslant-\lambda+\left(\frac{s}{\beta}+\frac{(a+b \beta) f}{h}-\frac{c l}{h}\right) x_{2}+\left(\frac{a+b \beta}{4 \beta^{2}}-\frac{f l}{h}\right) x_{2}^{2}+\frac{l x_{1} x_{2}}{\beta+x_{2}} \leqslant-\lambda+\frac{l x_{1} x_{2}}{\beta+x_{2}}
$$


Then compute

$$
L V_{2}=a x_{1}-b x_{1}^{2}-\frac{s c}{h} x_{2}-\frac{s f}{h} x_{2}^{2} \leqslant a x_{1}-b x_{1}^{2}-\frac{s f}{h} x_{2}^{2} .
$$

Hence

$$
L V(x) \leqslant M\left(-\lambda+\frac{l x_{1} x_{2}}{\beta+x_{2}}\right)+a x_{1}-b x_{1}^{2}-\frac{s f}{h} x_{2}^{2},
$$

where $M$ satisfies $M \lambda \geqslant a^{2} /(4 b)+2$. Now we are aimed to show

$$
L V(x) \leqslant-1 \text { for all } x \in \mathbb{R}_{+}^{2}-G:=G^{c} .
$$

As if this holds, let $x \in G^{c}$ be arbitrary and $\tau_{G}$ be the stopping time as defined in Lemma 5.1. From (5.3), we have

$$
0 \leqslant V\left(x_{0}\right)-\mathbb{E}_{x_{0}}\left(t \wedge \tau_{G} \wedge \tau_{k}\right), \quad \forall t>0 .
$$

Letting $k \rightarrow \infty$ and then $t \rightarrow \infty$, we have

$$
\mathbb{E}_{x_{0}}\left(\tau_{G}\right) \leqslant V\left(x_{0}\right), \quad \forall x_{0} \in G^{c}
$$

as required. To show that $(5.3)$ actually holds, we define

$$
G^{c}=G_{1}^{c} \cup G_{2}^{c} \cup G_{3}^{c} \cup G_{4}^{c},
$$

where

$$
\begin{array}{ll}
G_{1}^{c}=\left\{x \mid x_{1} \in\left(0, \epsilon_{1}\right]\right\} ; & G_{2}^{c}=\left\{x \mid x_{1} \in\left(0, \frac{1}{\epsilon_{1}}\right], x_{2} \in\left(0, \epsilon_{2}\right]\right\} ; \\
G_{3}^{c}=\left\{x \mid x_{1} \in\left[\frac{1}{\epsilon_{1}},+\infty\right)\right\} ; & G_{4}^{c}=\left\{x \mid x_{2} \in\left[\frac{1}{\epsilon_{2}},+\infty\right)\right\}
\end{array}
$$

with two constants $\epsilon_{1}, \epsilon_{2} \in(0,1)$ satisfying

$$
\epsilon_{1}^{2} \leqslant \frac{1}{M^{2} l^{2}} \bigwedge \frac{b}{2\left(N_{1}+1\right)}, \quad \epsilon_{2}^{2} \leqslant \frac{s f}{2 h\left(N_{2}+1\right)} \quad \text { and } \quad \epsilon_{2} \leqslant \frac{\beta \epsilon_{1}}{M l},
$$

where the constants $N_{1}$ and $N_{2}$ will be determined later. We then show that in any subset of $G^{c}$, (5.3) holds. From (5.4),

(a) if $x \in G_{1}^{c}$,

$$
L V(x) \leqslant-M \lambda+M l x_{1}+a x_{1}-b x_{1}^{2}-\frac{s f}{h} x_{2}^{2} \leqslant M l \epsilon_{1}-2 \leqslant-1 ;
$$


(b) if $x \in G_{2}^{c}$,

$$
L V(x) \leqslant-M \lambda+\frac{M l x_{1} x_{2}}{\beta}+a x_{1}-b x_{1}^{2}-\frac{s f}{h} x_{2}^{2} \leqslant \frac{M l \epsilon_{2}}{\beta \epsilon_{1}}-2 \leqslant-1 ;
$$

(c) if $x \in G_{3}^{c}$,

$$
L V(x) \leqslant-M \lambda+(M l+a) x_{1}-\frac{b x_{1}^{2}}{2}-\frac{b x_{1}^{2}}{2}-\frac{s f x_{2}^{2}}{h} .
$$

Note that the polynomial $-M \lambda+(M l+a) x_{1}-\frac{b x_{1}^{2}}{2}-\frac{s f x_{2}^{2}}{h}$ has an upper bound, say $N_{1}$. Hence

$$
L V(x) \leqslant N_{1}-\frac{b}{2 \epsilon_{1}^{2}} \leqslant-1
$$

(d) if $x \in G_{4}^{c}$,

$$
L V(x) \leqslant-M \lambda+(M l+a) x_{1}-b x_{1}^{2}-\frac{s f x_{2}^{2}}{2 h}-\frac{s f x_{2}^{2}}{2 h} .
$$

Note that the polynomial $-M \lambda+(M l+a) x_{1}-b x_{1}^{2}-\frac{s f x_{2}^{2}}{2 h}$ is again bounded, say by $N_{2}$, we have

$$
L V(x) \leqslant N_{2}-\frac{s f}{2 h \epsilon_{2}^{2}} \leqslant-1 .
$$

In all,

$$
L V(x) \leqslant-1 \text { for all } x \in G^{c} .
$$

Recall that Theorem 4.2 considers the dynamical behaviours of model (1.3) when $0<2 a<\sigma_{1}^{2}$ and $\sigma_{1}^{2}<2 a<\phi$. Next Theorem 5.2 shows that system (1.3) has a stationary distribution when $2 a>\frac{\phi}{1-\sigma_{2}^{2} /(2 h)-c / h}$. However we have not been able to prove the case when

$$
\phi<2 a<\frac{\phi}{1-\sigma_{2}^{2} /(2 h)-c / h} .
$$

Example 6.4 provides an illustration of it. 


\section{Examples and computer simulations}

We first give examples for SDE system (1.3) to die out.

Example 6.1. We perform a computer simulation of 10000 iterations of model (1.3) with initial value $x(0)=(1.0,0.1)^{T}$ using the Euler-Maruyama (EM) method [28] 32] with stepsize $\Delta=0.01$ and the system parameters given by

$$
a=1, b=0.1, s=6, \beta=5, h=0.9, c=2, f=0.5, \sigma_{1}=1.5 \text { and } \sigma_{2}=1.3 \text {. }
$$

It is easy to verify that these system parameters satisfy condition (4.2). By Theorem $4.2(a), x_{1}(t)$ and $x_{2}(t)$ will die out as $t \rightarrow \infty$ with probability 1 . The computer simulations shown in Figure $1(a)(b)$ support these results.

Example 6.2. We keep the system parameters the same as in Example 6.1 but let $\sigma_{1}=0.7$ instead. Obviously the system parameters obey condition (4.3). By Theorem $4.2(b)$,

$$
\lim _{t \rightarrow \infty} \frac{1}{t} \int_{0}^{t} x_{1}(u) d u=\frac{2 a-\sigma_{1}^{2}}{2 b}=7.55
$$

and $x_{2}(t)$ will become extinct as $t \rightarrow \infty$ with probability 1 . From Figure $1(c)(d)$, the prey abundance fluctuates around the level 7.55 while the consumer s die out. This is consistent with the result $s$ in Theorem 4.2 (b).

In Example 6.1 and 6.2, Remark 4.3 suggests that the solution of the deterministic model (1.2) has the property that $\lim _{t \rightarrow \infty} \frac{1}{t} \int_{0}^{t} \bar{x}_{1}(u) d u=\frac{a}{b}=10$ and the consumers tend to zero almost surely. Moreover, the situation where both species die out ultimately arised in model (1.3) does not happen in model (1.2). Figure 1(a)(b) support these results clearly. Then we study the case where the SDE system (1.3) has a stationary distribution.

Example 6.3. We keep the system parameters the same as in Example 6.1 but let $h=4, \sigma_{1}=0.1$ and $\sigma_{2}=0.2$. It is obvious that this group of parameters satisfies condition (5.1). From Theorem 5.2 both prey and predator populations have a stationary distribution. The ergodic property enables us to obtain the approximate probability distribution for the stationary distribution by computer simulation of a single sample path of a solution to model (1.3). Therefore the histogram of the 10000 iterations shown in Figure $2(b)(d)$ can be regarded as approximate p.d.f.s of the stationary distribution. On the other hand, as the parameters obey the condition $a>\frac{b \beta c}{h}$, there exists a positive equilibrium point $\bar{E}^{*}\left(\bar{x}_{1}^{*}, \bar{x}_{2}^{*}\right)$ of model (1.2). This is clearly shown in Figure 2 (a) (c). 
Example 6.4. We keep the system parameters the same as in Example 6.3 but let $c=3$, so that condition (5.5) is fulfilled. Figure $3(a)(c)$ indicates a stationary distribution of both species. If this is true, the histogram of the 10000 iterations shown in Figure $3(b)(d)$ can be regarded as approximate p.d.f.s of the stationary distribution.

\section{Discussion}

In this paper, the two-dimensional foraging arena model in presence of environmental perturbation is considered. After studying the existence and uniqueness of a positive solution to model (1.3), the long-time dynamical behaviours are generated. In order to investigate the effects of the environmental noises on the population dynamics, it is worth comparing the stochastic model (1.3) with the deterministic one (1.2). Firstly, the deterministic model $(1.2)$ has two nonnegative trivial equilibrium points $\bar{E}_{0}=(0,0)$ and $\bar{E}_{1}=\left(\frac{a}{b}, 0\right)$. Also under the condition $a>\frac{b \beta c}{h}$, there exists a positive equilibrium point $\bar{E}^{*}\left(\bar{x}_{1}^{*}, \bar{x}_{2}^{*}\right)$ which is also globally asymptotically stable. However, the stochastic model (1.3) only has one trivial equilibrium point $E_{0}=(0,0)$. Secondly, recall that both species of model (1.3) will die out ultimately if $2 a<\sigma_{1}^{2}$. In the real life, it may happen when a serious disease or severe weather occurs. However this situation does not happen in model (1.2). Figure 1(a)(b) shows this difference clearly. This suggests that the stochastic system is a more realistic model to describe the world than the deterministic one. Furthermore, recall that the deterministic model (1.2) will die out, in the sense that the prey population remains persistent while the consumers become extinct ultimately, provided that $a<\frac{b \beta c}{h}$. In the contrast, the stochastic model (1.3) goes to extinction even for some $a>\frac{b \beta c}{h}$ (but need to obey $2 a<\phi$ ) due to the presence of white noises. Hence we conclude that the presence of environmental noises brings a difference to the population dynamics.

Furthermore, we have shown the existence of a unique stationary distribution under condition (5.1). However, the dynamics of model (1.3) under (5.5) remain an open problem, though the computer simulation in Example 6.4 indicates a stationary distribution of both species. In the stochastic prey-predator model with Beddington-DeAngelis response [26], more restrictive conditions were produced to have a stationary distribution. These conditions were found based on the positive equilibrium of the corresponding deterministic system. From the model parameters point of view, however, it is not clear what cases have not been considered and hence need further investigation. 

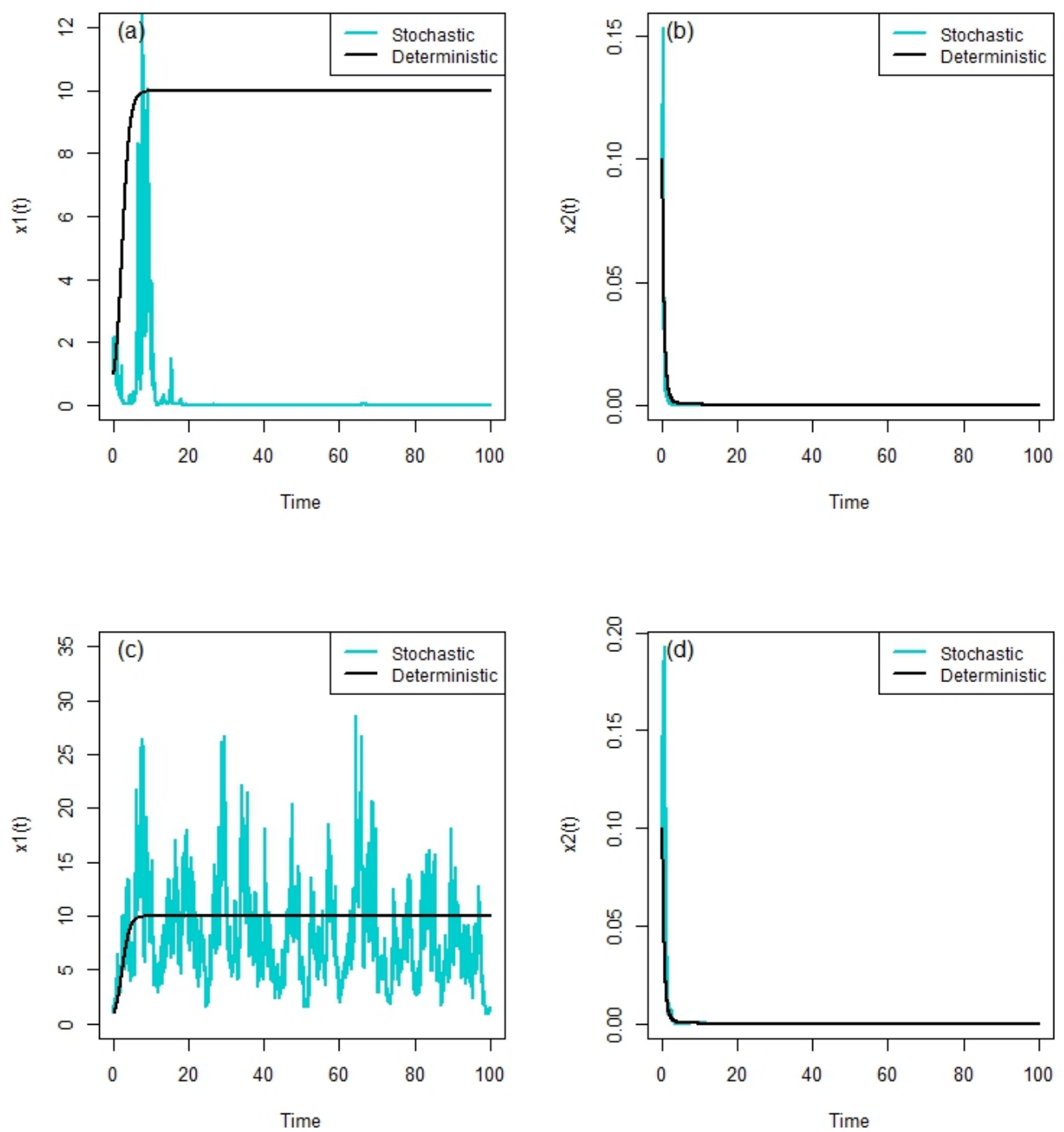

Figure 1: Computer simulations of the paths (a) $x_{1}(t)$ and (b) $x_{2}(t)$ of 10000 iterations of SDE model (1.3) using the EM scheme with stepsize $\Delta=0.01$ and initial value $x_{0}=(1.0,0.1)^{T}$ and the corresponding ODE paths (model (1.2)) with the system parameters provided by (6.1). Given the system parameters as in (6.1) except that $\sigma_{1}=0.7$, we get the computer simulation of paths (c) $x_{1}(t)$ and (d) $x_{2}(t)$ of 10000 iterations using the EM method with stepsize $\Delta=0.01$ and initial value $x_{0}=(1.0,0.1)^{T}$ and the corresponding ODE paths. 

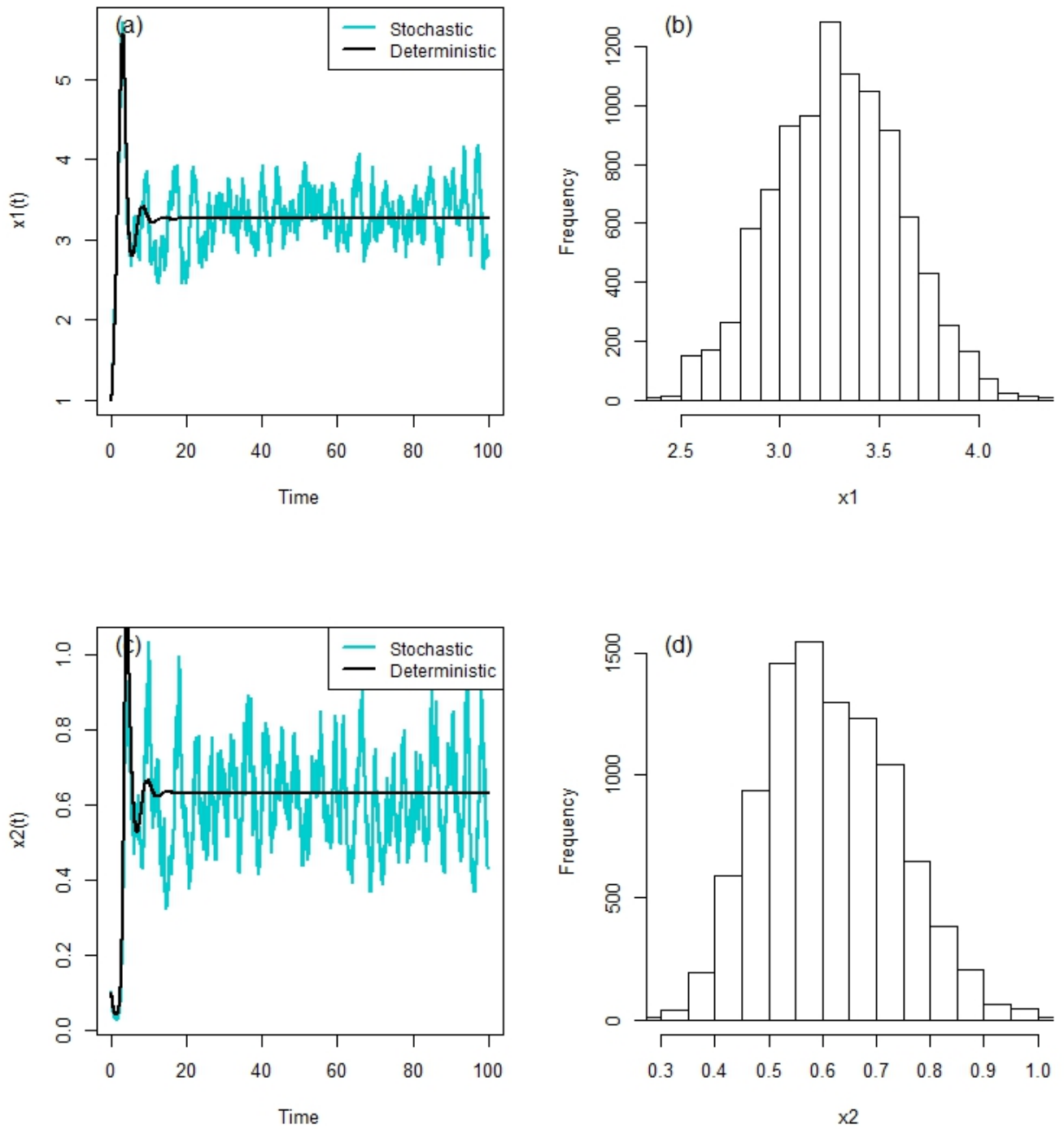

Figure 2: Computer simulation of the paths (a) $x_{1}(t)$ and (c) $x_{2}(t)$ of 10000 iterations of SDE model (1.3) using the EM technique with stepsize $\Delta=0.01$ and initial values $x_{0}=(1.0,0.1)^{T}$ and the corresponding ODE paths (model (1.2) with the parameters provided by 6.1 except that $h=4, \sigma_{1}=0.1$ and $\sigma_{2}=0.2$, followed by the histograms of the SDE paths (b) $x_{1}(t)$ and (d) $x_{2}(t)$. 

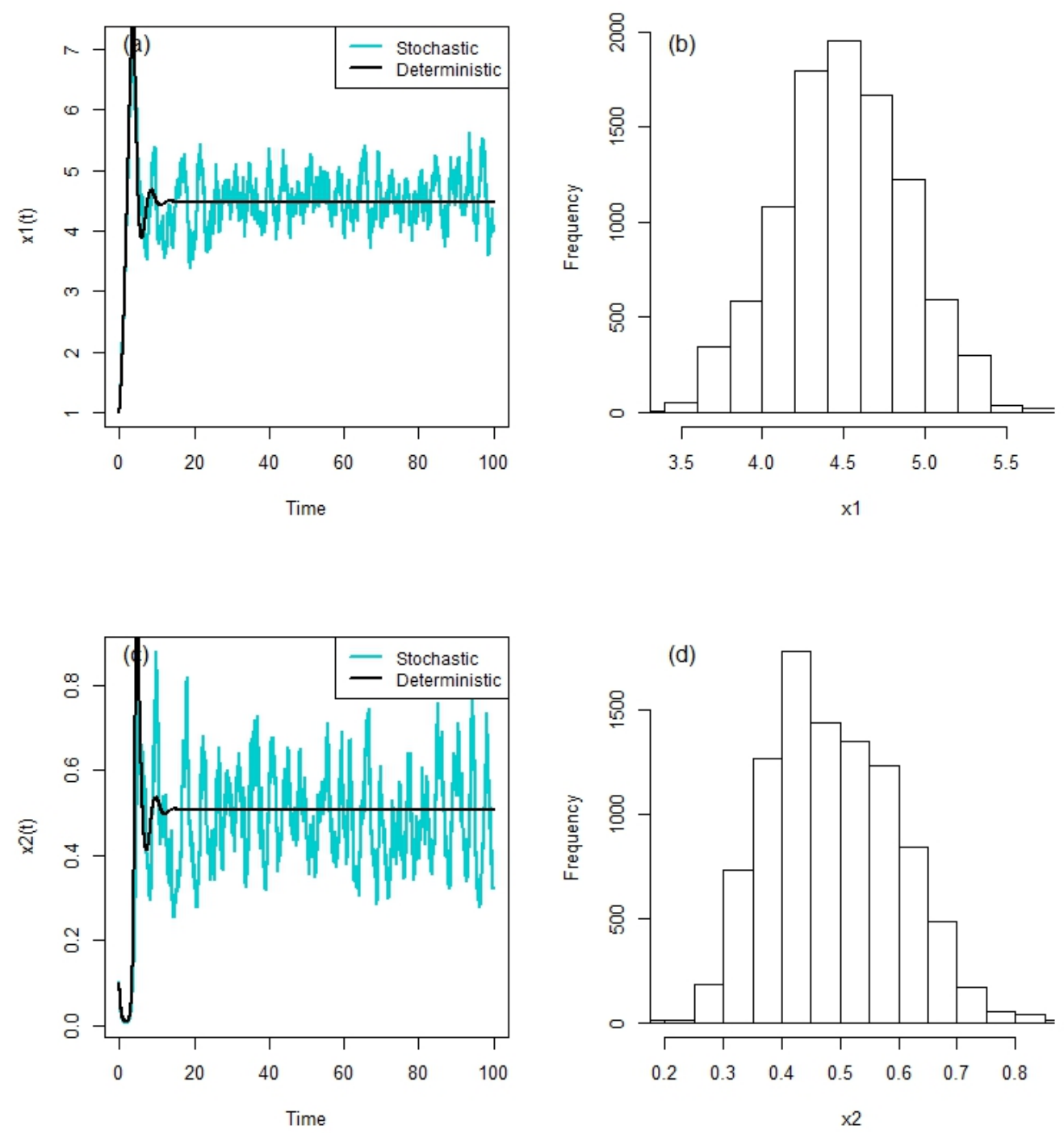

Figure 3: Computer simulation of the paths (a) $x_{1}(t)$ and (c) $x_{2}(t)$ of 10000 iterations of SDE model (1.3) using the EM technique with stepsize $\Delta=0.01$ and initial values $x_{0}=(1.0,0.1)^{T}$ and the corresponding ODE paths (model (1.2)) with the parameters the same as in Example 6.3 except that $c=3$, followed by the histograms of the SDE paths (b) $x_{1}(t)$ and (d) $x_{2}(t)$. 
For the population system having a stationary distribution, the ergodic formula enables us to obtain the approximate probability distribution by computer simulation of a single sample path of a solution to the SDE model, although the mean value and variance have not been explicitly computed like in [29, 31]. In this paper, model (1.3) considers the noise perturbation on the intrinsic growth rate of prey and the density-dependent death rate of consumers. However stochastically perturbating other parameters such as the capturing rate of predators and quadratic mortality rates of both species is also an interesting topic and will be investigated in the future.

\section{Acknowledgements}

The authors would like to thank the reviewers and the editors for their very professional comments and suggestions. The authors would like to thank the Leverhulme Trust (RF-2015-385), the Royal Society (WM160014, Royal Society Wolfson Research Merit Award), the Royal Society and the Newton Fund (NA160317, Royal Society-Newton Advanced Fellowship) and the EPSRC (EP/K503174/1) for their financial support. The first author would also like to thank China Scholarship Council, the MASTS pooling initiative (The Marine Alliance for Science and Technology for Scotland) and the University of Strathclyde for their financial support. MASTS is funded by the Scottish Funding Council (HR09011).

\section{References}

[1] H. R. Akcakaya, R. Arditi, L. R. Ginzburg, Ratio-dependent predation: an abstraction that works, Ecology 76 (3) (1995) 995-1004. doi:10.2307/ 1939362 .

[2] M. R. Heath, D. C. Speirs, J. H. Steele, Understanding patterns and processes in models of trophic cascades, Ecology Letters 17 (1) (2014) 101-114. doi : 10.1111/ele.12200. URL http://doi.wiley.com/10.1111/ele.12200

[3] C. S. Holling, Some characteristics of simple types of predation and parasitism, The Canadian Entomologist 91 (7) (1959) 385-398.

[4] W. Gentleman, A. Leising, B. Frost, S. Strom, J. Murray, Functional responses for zooplankton feeding on multiple resources: a review of assumptions and biological dynamics, Deep Sea Research Part II: Topical Studies 
in Oceanography 50 (22) (2003) 2847-2875. doi:10.1016/j.dsr2.2003. 07.001

[5] R. Arditi, L. R. Ginzburg, Coupling in predator-prey dynamics: ratiodependence, Journal of theoretical biology 139 (3) (1989) 311-326.

[6] R. Arditi, L. R. Ginzburg, How Species Interact: Altering the Standard View on Trophic Ecology, Vol. NY,204pp, Oxford University Press, 2012.

[7] P. A. Abrams, The fallacies of" ratio-dependent" predation, Ecology 75 (6) (1994) 1842-1850. doi:10.2307/1939644.

[8] J. R. Beddington, Mutual interference between parasites or predators and its effect on searching efficiency, The Journal of Animal Ecology (1975) 331340 .

[9] D. L. DeAngelis, R. Goldstein, R. O'neill, A model for trophic interaction, Ecology 56 (4) (1975) 881-892. doi : 10.2307/1936298.

[10] S. Khajanchi, Dynamic behavior of a Beddington-DeAngelis type stage structured predator-prey model, Applied Mathematics and Computation 244 (2014) 344-360.

[11] C. Walters, D. Pauly, V. Christensen, J. F. Kitchell, Representing density dependent consequences of life history strategies in aquatic ecosystems: EcoSim II, Ecosystems 3 (1) (2000) 70-83.

[12] R. N. Ahrens, C. J. Walters, V. Christensen, Foraging arena theory, Fish and Fisheries 13 (1) (2012) 41-59. doi:10.1111/j.1467-2979.2011.00432. $\mathrm{X}$.

[13] H. Li, Y. Takeuchi, Dynamics of the density dependent predator-prey system with Beddington-DeAngelis functional response, Journal of Mathematical Analysis and Applications 374 (2) (2011) 644-654.

[14] W. Gurney, R. M. Nisbet, Ecological Dynamics, Oxford University Press, 1998.

[15] X. Mao, G. Marion, E. Renshaw, Environmental Brownian noise suppresses explosions in population dynamics, Stochastic Processes and their Applications 97 (1) (2002) 95-110. doi:10.1016/S0304-4149(01)00126-0. 
[16] P. Cattiaux, S. Méléard, Competitive or weak cooperative stochastic LotkaVolterra systems conditioned on non-extinction, Journal of mathematical biology 60 (6) (2010) 797-829.

[17] X. Mao, S. Sabanis, E. Renshaw, Asymptotic behaviour of the stochastic Lotka-Volterra model, Journal of Mathematical Analysis and Applications 287 (1) (2003) 141-156. doi:10.1016/S0022-247X (03) 00539-0.

[18] A. Bahar, X. Mao, Stochastic delay Lotka-Volterra model, Journal of Mathematical Analysis and Applications 292 (2) (2004) 364-380. doi:10.1016/ j.jmaa.2003.12.004.

[19] X. Mao, C. Yuan, J. Zou, Stochastic differential delay equations of population dynamics, Journal of Mathematical Analysis and Applications 304 (1) (2005) 296-320. doi:10.1016/j.jmaa.2004.09.027.

[20] Y. Takeuchi, N. Du, N. Hieu, K. Sato, Evolution of predator-prey systems described by a Lotka-Volterra equation under random environment, Journal of Mathematical Analysis and Applications 323 (2) (2006) 938-957. doi: 10.1016/j.jmaa.2005.11.009

[21] C. Ji, D. Jiang, N. Shi, Analysis of a predator-prey model with modified Leslie-Gower and Holling-type II schemes with stochastic perturbation, Journal of Mathematical Analysis and Applications 359 (2) (2009) 482-498. doi:10.1016/j.jmaa.2009.05.039.

[22] J. Lv, K. Wang, Asymptotic properties of a stochastic predator-prey system with Holling II functional response, Communications in Nonlinear Science and Numerical Simulation 16 (10) (2011) 4037-4048. doi:10.1016/j. cnsns.2011.01.015.

[23] Q. Liu, L. Zu, D. Jiang, Dynamics of stochastic predator-prey models with Holling II functional response, Communications in Nonlinear Science and Numerical Simulation 37 (2016) 62-76. doi:10.1016/j.cnsns.2016. 01.005

[24] X. Zhang, Y. Li, D. Jiang, Dynamics of a stochastic Holling type II predatorprey model with hyperbolic mortality, Nonlinear Dynamics 87 (3) (2017) 2011-2020. 
[25] C. Ji, D. Jiang, X. Li, Qualitative analysis of a stochastic ratio-dependent predator-prey system, Journal of Computational and Applied Mathematics 235 (5) (2011) 1326-1341. doi:10.1016/j.cam.2010.08.021.

[26] C. Ji, D. Jiang, Dynamics of a stochastic density dependent predator-prey system with Beddington-DeAngelis functional response, Journal of Mathematical Analysis and Applications 381 (1) (2011) 441-453. doi:10.1016/ j.jmaa.2011.02.037.

[27] X. Mao, C. Yuan, Stochastic differential equations with Markovian switching, Imperial College Press, 2006.

[28] X. Mao, Stochastic differential equations and applications, Elsevier, 2007.

[29] A. Gray, D. Greenhalgh, L. Hu, X. Mao, J. Pan, A stochastic differential equation SIS epidemic model, SIAM Journal on Applied Mathematics 71 (3) (2011) 876-902.

[30] R. Khasminskii, Stochastic stability of differential equations, Vol. 66, Springer Science \& Business Media, 2011.

[31] X. Mao, Stationary distribution of stochastic population systems, Systems \& Control Letters 60 (6) (2011) 398-405. doi:10.1016/j . sysconle. 2011. 02.013

[32] D. J. Higham, An algorithmic introduction to numerical simulation of stochastic differential equations, SIAM review 43 (3) (2001) 525-546. doi : $10.1137 / \mathrm{S} 0036144500378302$. 